\title{
Soft Glass Based Large Mode Area Photonic Bandgap Fibre for Mid-Infrared Applications
}

\author{
Somnath Ghosh ${ }^{1}$, Sonali Dasgupta ${ }^{2 *}$, Francesco Poletti ${ }^{2}$, Ravi Varshney ${ }^{1}$, Bishnu P. Pal $^{1}$, David. J. \\ Richardson $^{2}$ \\ ${ }^{1}$ Indian Institute of Technology Delhi, Hauz Khas, New Delhi - 110016, India \\ ${ }^{2}$ Optoelectronics Research Centre, University of Southampton, United Kingdom SO167FB \\ *sxd@orc.soton.ac.uk
}

\begin{abstract}
An all-solid LMA Bragg fibre (mode area exceeding $1100 \mu \mathrm{m}^{2}$ ) is presented for midinfrared applications, based on a new design strategy that induces large differential loss between fundamental and higher order modes for effective single-mode operation.

OCIS codes: (060.4005) Microstructured fibres (060.2280) Fibre design and fabrication
\end{abstract}

\begin{abstract}
1. Introduction
The mid-infrared (IR) wavelengths $(2-25 \mu \mathrm{m})$ have recently become increasingly important due to emerging applications in areas such as mid-IR spectroscopy for astronomy, optical coherence tomography, and chemical sensing. This has spawned wide interest in the development of optical fibres that can efficiently guide light and enable high power laser delivery at these wavelengths. Besides the choice of optimum material system, a major challenge in this field is the design of optical fibres that enable efficient distortion-free transmission at high power levels and supplement the design of efficient mid-IR fibre sources. Single-mode large-mode area (LMA) fibres are crucial in this regard because they mitigate the undesirable nonlinear processes (e.g. stimulated Brillouin scattering, stimulated Raman scattering, four-wave mixing) in the fibre that deteriorate their power handling capability.

Single-mode LMA fibres based on step index profiles are limited in functionality due to their high bend-loss sensitivity and tight tolerances in fabrication parameters. Thus, more recently, alternative routes have been explored, which have relied on the use of multimode LMA fibres in which effective guidance of the fundamental mode (FM) is achieved by inducing a large differential loss for its higher order modes (HOMs). The necessary differential losses can be engineered through fibre design by a number of means including controlling the inherent modal confinement loss and/or relative bend loss. Techniques that maximize the coupling efficiency into the FM are also critical. Most of these fibre designs are based on microstructured optical fibres (MOFs) that provide unprecedented structural design freedom for controlling their propagation characteristics. In this context, all-solid Bragg fibres are extremely promising as they enable better control over the structural parameters during fibre drawing thereby ensuring good repeatability with minimal transverse deformations and allowing for accurate targeting of the optical characteristics in the final fibre [1,2]. Bragg fibres can also provide considerably lower bending losses as compared to MOFs (with air holes) with the same size of the mode field [3].

The highly nonlinear non-silica based glasses are promising candidates for fabricating the mid-IR fibres owing to their good transparency across the mid-IR wavelength spectrum [4]. Due to their lower toxicity, higher thermal stability, and mature fabrication process, the lead-silicate glasses are attractive for fabrication of fibres and fibrebased devices operating in the wavelength range of $2-5 \mu \mathrm{m}$ [5]. In this paper, we report a new design strategy to realize a soft glass (lead silicate) based all-solid Bragg fibre with effective mode area exceeding $1100 \mu \mathrm{m}^{2}$ across the operating wavelength range of $2-4 \mu \mathrm{m}$. The proposed design route enables us to realize differential losses as large as three orders of magnitude between the FM and HOMs, meaning that fibre lengths of just few tens of centimetres are sufficient to provide an effective single-mode output with a large mode area.
\end{abstract}

\section{Fibre design}

It is well known that a Bragg fibre with a finite cladding is a leaky optical structure [6]. Through appropriate design, this feature has been gainfully exploited to lower the loss of the FM while allowing the HOMs to exhibit larger losses [7]. The loss of the first higher order $\mathrm{LP}_{11}$ mode usually determines the length of fibre required to make it effectively single-moded. The losses of the other core-guided HOMs are typically orders of magnitude higher and hence, they are lost from the core within a short length of the fibre. In the case of a LMA fibre, a large core diameter is a pre-requisite. However, large cores inevitably support more unwanted HOMs, which ordinarily leads to poor mode quality of the output beam. Here, we propose a novel design strategy wherein rather than following the commonly used low-loss criterion (for the FM) for designing the cladding layers [6]; we choose the layers so that they are anti-resonant for the $\mathrm{LP}_{11}$ mode while simultaneously reflecting the FM back constructively into the core. The HOMs extend more into the cladding as compared to the FM and hence, the effect of the anti-resonant cladding 
on the HOMs is much stronger. In addition to the cladding, we design our fibre core so that it is anti-resonant for the first higher order $\mathrm{LP}_{11}$ mode. This increases its confinement loss although having a negligible impact on the confinement of the FM. Table 1 shows the phase accumulated by the $\mathrm{LP}_{01}$ and the $\mathrm{LP}_{11}$ modes in the core and the cladding, and demonstrates how the choice of the core and cladding widths preferentially leaks away the HOMs. In the wave-vector space, the choice of the core and cladding dimensions ensures that the transverse wave vector of the FM still lies well within the photonic bandgap while the wave vector of the $\mathrm{LP}_{11}$ mode and other HOMs lie close to the band edge. The material system chosen is based on two thermally compatible lead-silicate glasses: LLF1 (refractive index $n_{1}=1.5232$ ) and SF6 (refractive index $n_{2}=1.7577$ ) (at the wavelength of $2 \mu \mathrm{m}$ ). The state-of-theart extrusion-based fibre fabrication technique for these glasses is mature enough to realize the pre-determined set of structural parameters to obtain the desired optical characteristics [5].

Table1. Phase accumulated by the $\mathrm{LP}_{01}$ and $\mathrm{LP}_{11}$ modes in the designed Bragg fibre

\begin{tabular}{|c|c|c|}
\hline & $\begin{array}{c}\mathrm{LP}_{01} \\
\text { (in units of } \pi \text { ) }\end{array}$ & $\begin{array}{c}\mathrm{LP}_{11} \\
\text { (in units of } \pi \text { ) }\end{array}$ \\
\hline$k_{c} r_{c}$ & 0.670 & 1.000 \\
\hline$k_{1} d_{1}$ & 0.530 & 0.795 \\
\hline$k_{2} d_{2}$ & 0.965 & 0.965 \\
\hline
\end{tabular}

where $k_{j}$ is defined as $k_{j}^{2}=k_{0}^{2}\left(n_{j}^{2}-n_{\text {eff }}^{2}\right), j=1,2$ and $k_{c}=k_{2} ; n_{\text {eff }}$ is the effective index of the $\mathrm{LP}_{01}$ or the $\mathrm{LP}_{11}$ mode.

\section{Results and discussions}

Fig.1 shows the refractive index profile of the designed fibre, which comprises of two cladding bi-layers. The outermost layer is assumed to be a high-index layer. Fig. 2 shows the calculated confinement loss of the $\mathrm{LP}_{01}$ and the next higher order $\mathrm{LP}_{11}$ mode. It clearly demonstrates that a differential loss spanning more than three orders of magnitude across the entire operating wavelength range of $2-4 \mu \mathrm{m}$ can be achieved.

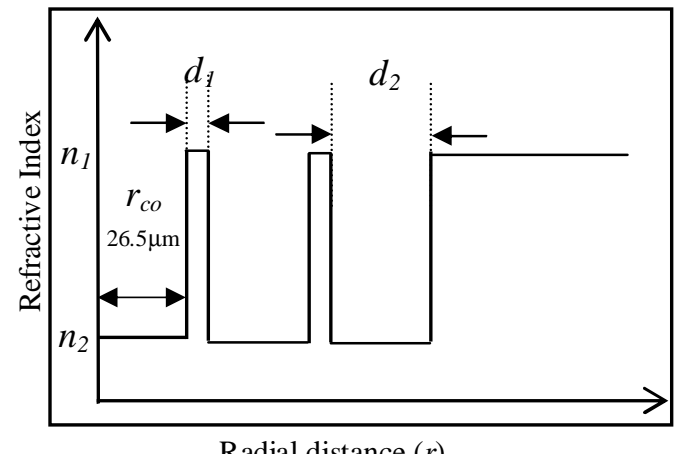

Radial distance $(r)$

Fig. 1. Refractive index profile of the proposed LMA Bragg fibre.

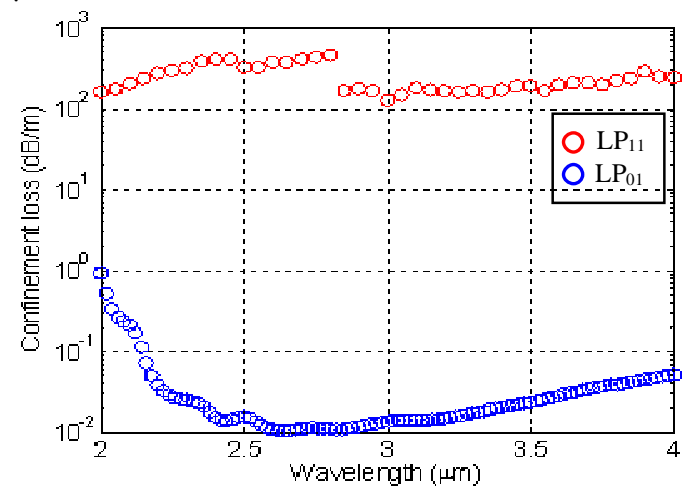

Fig. 2. Differential confinement loss between the FM and $\mathrm{LP}_{11}$ mode of the proposed fibre.

Fig. 3 shows the effective area variation of the FM across this wavelength range, with an average area of $1100 \mu \mathrm{m}^{2}$. Note that the variation is almost negligible across a broad wavelength range. Thus, the proposed fibre of length $\sim 25 \mathrm{~cm}$ should be suitable for effectively single-mode high power delivery at wavelengths between $\sim 2-4 \mu \mathrm{m}$. The higher nonlinearity of the chosen lead silicate glasses implies that the effective nonlinearity of the proposed LMA fibre is $\sim 0.5(\mathrm{~W} . \mathrm{Km})^{-1}$; which is comparable to a standard silica fibre with a much smaller geometric mode area. Figure 4 shows the dispersion spectrum of the fibre. The zero dispersion wavelength (ZDW) is at $\sim 2.04 \mu \mathrm{m}$ and the dispersion slope at the centre of the operating wavelength window (at $3 \mu \mathrm{m})$ is $\sim 0.07 \mathrm{ps} /\left(\mathrm{km} . \mathrm{nm}^{2}\right)$. Interestingly, these dispersion properties are ideal for pumping the fibre with Thulium/ holmium doped fibre lasers. Thus, in conjunction with the large effective area (that exhibits negligible variation with wavelength) and the high nonlinearity inherent of the soft glasses, the fibre could also be used for high power supercontinuum (SC) generation across the $2-4 \mu \mathrm{m}$ wavelength range. High power SC generation is difficult to achieve with small core fibers due to the limitations imposed by the damage threshold of the fiber material.

It is important to note that currently the material loss of the silicate glasses is limited by the purity of the glasses and stands at $\sim 2 \mathrm{~dB} / \mathrm{m}$ across the $2-4 \mu \mathrm{m}$ wavelength range. Thus, in practice, this material loss would dominate the total 


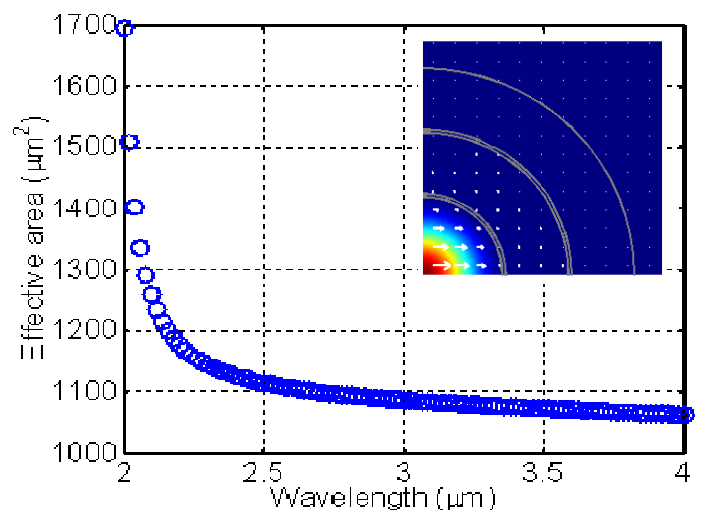

Fig. 3. Variation of effective area of the FM with wavelength. Inset shows the intensity profile of the FM in one-quarter of the fibre structure at $2.1 \mu \mathrm{m}$ wavelength. The grey lines indicate the fiber geometry. Arrows indicate the polarization state of the electric field.

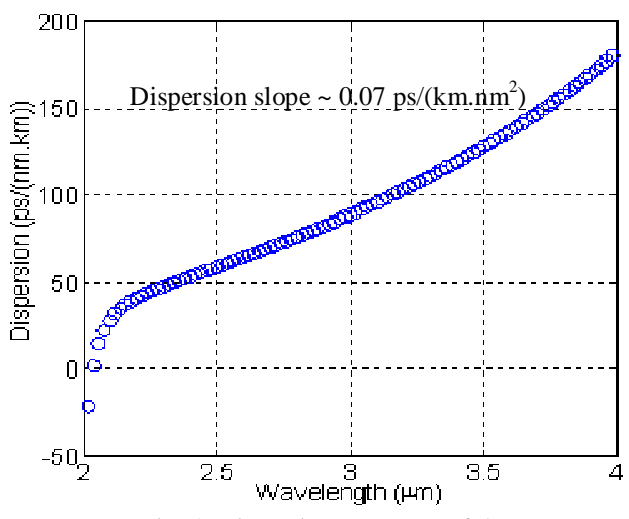

Fig. 4. Dispersion spectrum of the FM

loss of the FM and any other HOMs in the fibre that exhibit confinement loss less than the material loss. The proposed fibre does support such low loss modes but detailed investigations show that none of these modes are coreguided modes. Rather they are the modes supported by the high-index rings and hence, a selective launch can easily prevent them from being excited. We would also like to point out that the core of the designed fibre supports the $\mathrm{LP}_{02}$ mode which exhibits an average confinement loss of $\sim 1 \mathrm{~dB} / \mathrm{m}$ across the operating wavelength range. Since this mode has the same symmetry as the FM, it would definitely be excited when we launch the FM, thereby making the fibre dual-moded. However, interestingly, the loss of this mode is still two orders of magnitude higher than that of the FM. Hence, with improvements in glass purity and the bulk loss of the silicate glasses expected to go down to $\sim 0.1 \mathrm{~dB} / \mathrm{m}$ in the near future, the differential loss between the FM and the higher order $\mathrm{LP}_{11}$ and $\mathrm{LP}_{02}$ modes is still sufficient to enable modal discrimination of greater than $20 \mathrm{~dB}$ in less than a meter length of the fibre, allowing effectively single-mode guidance. The discrimination could be further improved by exploiting the higher sensitivity of the HOMs through bending. We also investigated the tolerance performance of the proposed fibre design with respect to variations in the cladding layer thicknesses. It is observed that variations of up to $\pm 5 \%$ in the cladding layer thickness do not significantly alter the propagation characteristics of the relevant modes.

\section{Conclusions}

In conclusion, we have presented a novel design route to enable large modal discrimination between the FM and the HOMs in a soft glass-based photonic bandgap LMA fibre. We have shown that few tens of centimeters length of the proposed fibre would be sufficient for achieving effective single-mode guidance across the $2-4 \mu \mathrm{m}$ wavelength range with an effective area of the FM exceeding $1100 \mu \mathrm{m}^{2}$. Combined with the zero dispersion wavelength at $2.04 \mu \mathrm{m}$ which is ideal for SC-pumping with high-power Thulium-doped lasers, the proposed fibre is a promising candidate for both high power SC generation and high power delivery applications at mid-infrared wavelengths.

\section{Acknowledgements}

This work is supported by the ongoing UK-India Education and Research Initiative (UKIERI) collaboration project. SG gratefully acknowledges his Pre-doctoral graduate student level exchange visit to the ORC under this project.

\section{References}

1. X. Feng, F. Poletti, A. Camerlingo, F. Parmigiani, P. Horak, P. Petropoulos, W.H. Loh, D.J. Richardson, "Dispersion-shifted all-solid high index-contrast microstructured optical fibre for nonlinear applications at 1.55 $\mu \mathrm{m}$," Opt. Express 17, 20249 (2009).

2. S. Dasgupta, B.P. Pal, and M.R. Shenoy, "Nonlinear Spectral Broadening in Solid-Core Bragg Fibres," J. Lightwave Technol. 25, 2475 (2007).

3. M.E. Likhachev, S.L. Semjonov, M.M. Bubnov, E.M. Dianov, V.F. Khopin, M.Yu. Salganskii, M.A. Gurjanov, A.N. Gurjanov, R.Jamier, P.Viale, S.Fevrier, J.M. Blondy, "Development and study of Bragg fibres with a large mode field and low optical losses" Quan. Electron., 36, 581 (2006).

4. X. Feng, A.K. Mairaj, D.W. Hewak, and T.M. Monro, "Nonsilica Glasses for Holey Fibres", J. of Lightwave Technol., 23, 6, (2005).

5. X. Feng, T.M. Monro, P. Petropoulos, V. Finazzi, and D.J. Richardson, "Extruded single mode high-index core one-dimensional microstructured optical fibre with high index contrast for highly nonlinear optical deivces", App. Phys. Lett., 87, 081110 (2005).

6. P. Yeh, A. Yariv, and E. Marom, "Theory of Bragg Fibre”, J. Opt. Soc. A., 68, 1196 (1978).

7. S. Février, R. Jamier, J.M. Blondy, S.L. Semjonov, M.E. Likhachev, M.M. Bubnov, E.M. Dianov, V.F. Khopin, M.Y. Salganskii and A.N. Guryanov, "Low-loss singlemode large area all-silica photonic bandgap fibre", Opt. Express, 14, 2 (2006). 